\title{
Identificación de variantes genéticas asociadas a hipercolesterolemia familiar en niños y adolescentes de la Región del Biobío, Chile
}

\author{
ANDREA SÁNCHEZ ${ }^{1, \mathrm{a}}$, PAULINA BUSTOS ${ }^{1, \mathrm{~b}}$, PAULA HONORATO ${ }^{1, \mathrm{~b}}$, \\ KATIA SÁEZ ${ }^{2, \mathrm{a}}$, CINTHIA ELIM-JANNES ${ }^{3, \mathrm{a}}$, NATALIA BARRIGA ${ }^{1, \mathrm{c}}$, \\ GUILLERMO IBIETA ${ }^{4}$, LUIS PÉREZ ${ }^{4}$, RODRIGO ALONSO ${ }^{5}$, \\ CLAUDIA RADOJKOVIC ${ }^{1, \mathrm{~A}}$, SYLVIA ASENJO
}

\section{Identification of genetic variants associated with familial hypercholesterolemia in Chilean children and adolescents}

Background: Familial hypercholesterolemia (FH) is commonly associated with mutations in $=L D L$ receptor (LDLR), apolipoprotein $B(A P O B)$ and proprotein convertase subtilisin/kexin type 9 (PCSK9). Aim: To identify genetic variants associated with $\mathrm{FH}$ in a population of children and adolescents with hypercholesterolemia or a family history of-demonstrated early CVD. Material and Methods: Clinical and biochemical parameters were evaluated, and nine genes related to FH were sequenced namely $L D L R$, APOB, PCSK9, LDLRAP1, LIPA, APOE, ABCG5, ABCG8 and STAP1, in 55 children and adolescents aged 1 to 18 years old, from non-consanguineous families. Results: Mutations associated with FH were found in 17 children and adolescents, corresponding to p.Asp47Asn, duplication of exons 13-15 and p.Ser326Cys of the LDLR gene;-p.Glu204* and Ile268Met of the APOE gene. Thirteen patients were heterozygous, two homozygous, two compound heterozygous, and one double heterozygous. Conclusions: Children and adolescents carrying mutations associated with $\mathrm{FH}$ were found by selective screening, which constitutes the first stage in the identification of genetic variants in our country.

(Rev Med Chile 2021; 149: 1267-1274)

Key words: Apolipoprotein E; Hyperlipoproteinemia Type II; Receptors, $L D L$.

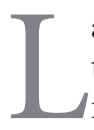

a Hipercolesterolemia Familiar (HF) es un transtorno genético frecuente, de transImisión autosómico dominante, que se caracteriza por niveles plasmáticos elevados de colesterol asociado a lipoproteinas de baja densidad (C-LDL) desde el nacimiento y un riesgo elevado de desarrollar enfermedad cardiovascular (ECV) ateroesclerótica prematura ${ }^{1-3}$. La HF es causada principalmente por variantes en tres



Trabajo financiado por Fondos internos de investigación Universidad de Concepción: Proyecto VRIDUDEC N $\mathrm{N}^{\circ} 218.088 .028-1.0 \mathrm{y}$ NN 219.072.041-M.

Los autores declaran no tener conflictos de interés.

Recibido el 18 de febrero de 2021 , aceptado el 25 de mayo de 2021.

Correspondencia a:

Andrea Sánchez

Departamento de Bioquímica Clínica e Inmunología, Facultad de Farmacia, Universidad de Concepción, Chile. andrsanc@udec.cl

genes codificantes para proteínas fundamentales en el metabolismo lipídico, tales como, el receptor de LDL ( $R L D L)$ (70-90\% de los casos), el gen de apolipoproteina B $(A P O B)(\sim 5 \%)$ y la proproteina convertasa de subtilisina/kexina tipo 9 (PCSK9 $)^{4-7}$. Muy raramente, mutaciones en otros genes involucrados en el metabolismo de las lipoproteínas como son el gen que codifica la apolipoproteina $\mathrm{E}(A P O E)$ y el de la proteína 
adaptadora transductora de señal (STAP1) pueden causar un fenotipo similar a HF .

La prevalencia estimada actual de la HF heterocigota es de 1 en 250 individuos de la población general, que aumenta considerablemente en sujetos con ECV prematura ${ }^{2,7-10}$. Por otra parte, la HF Homocigota en una entidad rara, con una prevalencia estimada en 1:450.000 individuos en población general ${ }^{3}$. Por tanto, en Chile se estiman en más de 70.000 los casos con HF heterocigota y en 40 los casos con HF homocigota.

En Chile no existen programas establecidos para la detección de la HF, si bien en la orientación técnica del Minsal 2018 se hace mención a la importancia del diagnóstico temprano y tratamiento oportuno para prevenir el desarrollo de la enfermedad CV. Se han descrito en los últimos años las características moleculares en sujetos heterocigotos y homocigotos en conjunto con otros países que conforman la Red Iberoamericana de Hipercolesterolemia Familiar ${ }^{11,12}$.

Con respecto a las variantes descritas, se ha señalado que Chile comparte con España alguna de las mutaciones más comunes en el gen del $R L D L$, las cuales corresponden a p.Asp221Gly, p.Gln92Glu y p.Gly592Glu, y con Brasil la mutación p.Ser326Cys ${ }^{11}$. En cuanto a las mutaciones en el gen de la $A p o B$, se ha identificado en Chile la mutación p.Arg3527Gln, descrita igualmente en España, Brasil, México y otros países de América Latina ${ }^{11}$.

El objetivo de este estudio fue identificar la presencia de variantes genéticas asociadas a HF para el apoyo del diagnóstico en una población de niños y adolescentes portadores de hipercolesterolemia y/o antecedentes familiares de ECV precoz demostrada.

\section{Materiales y Métodos}

\section{Población de estudio y criterios de inclusión}

Estudio descriptivo de corte transversal de una población de niños y adolescentes con edades comprendidas entre 1 y 18 años, todos ellos casos índices independientes de familias no consanguíneas con sospecha diagnóstica de HF de la región del Biobío. El reclutamiento comprendió un período de dos años y se realizó: 1) mediante pesquisa selectiva en hijos de padres con ECV precoz demostrada y atendidos en el Laboratorio de Hemodinamia del Hospital Guillermo Grant Benavente. La ECV precoz demostrada se define como infarto agudo al miocardio y/o revascularización coronaria percutánea o quirúrgica en hombres menores de 55 años y mujeres menores de 60 años; y, 2) en niños y adolescentes referidos al Policlínico de Endocrinología por presentar dislipidemia.

El diagnóstico de sospecha de HF se basó en los siguientes criterios: la presencia de C-LDL $\geq 190$ $\mathrm{mg} / \mathrm{dL} \mathrm{o} \geq 150 \mathrm{mg} / \mathrm{dL}$ con antecedentes familiares de hipercolesterolemia y/o ECV precoz demostrada en un familiar de primer grado y/o presencia de xantomas tendinosos y cutáneos, xantelasma o arco corneal ${ }^{2}$.

Los padres o tutores de niños y adolescentes que aceptaron participar de este estudio firmaron un consentimiento informado y los mayores de 10 años firmaron el asentimiento. Este estudio respetó las normas éticas establecidas en la Declaración de Helsinki y cuenta con la aprobación del Comité Ético-Científico del Servicio de Salud Concepción (Proyecto VRID-UDEC No 218.088.028-1.0; No 219.072.041-M)

\section{Análisis antropométricos}

Los escolares participantes fueron medidos en posición vertical con estadiómetro de precisión $0,1 \mathrm{~cm}$ y pesados en balanza TANITA. Se calculó el índice de masa corporal (IMC) $\left(\mathrm{kg} / \mathrm{m}^{2}\right)$, su percentil de acuerdo con tablas del $\mathrm{CDC}^{13}$.

\section{Análisis bioquímicos}

En la población en estudio se colectó una muestra de sangre periférica después de 12 horas de ayuno, La concentración de colesterol total (CT), colesterol-HDL (C-HDL) y triglicéridos (TG) se determinaron en un analizador clínico automatizado (SIEMENS). Los niveles de C-LDL se calcularon mediante la fórmula de Friedewald si los triglicéridos eran inferiores a $350 \mathrm{mg} / \mathrm{dL}^{14}$.

\section{Análisis molecular}

El ADN genómico se obtuvo de la capa leucocitaria con columnas de purificación (QIAGEN) según las instrucciones del fabricante, el cual fue secuenciado para 5 genes relacionados a HF autosómica dominante (LDLR, APOB, PCSK9, $A P O E$ y $S T A P 1)$ y 1 gen a HF autosómica recesiva (LDLR adaptor protein 1 (LDLRAP1)). Además, 
con el objetivo de realizar el diagnóstico diferencial de dos patologías que cursan con un fenotipo similar a HF, sitosterolemia y la enfermedad por depósitos de ésteres de colesterol, se secuenciaron los genes ATP-binding casette subfamily $G$ member 5 y 8 (ABCG5, ABCG8) y lipasa ácida lisosomal (LIPA). Los templados se prepararon en el Sistema One Touch y secuenciados en la plataforma Ion Torrent PGM ${ }^{\circledR}$ (Thermofisher) en un 316v2 Ion Chip. Los análisis bioinformáticos se realizaron en CLC Genomics Workbench 9,5 (QIAGEN). Después de filtrar con poblaciones de control (NHLBI-ESP6500, AbraOM, ExAC y gnomAD), fueron consultadas todas las mutaciones potenciales para su descripción previa en ClinVar, Base de Datos de Mutaciones del Genoma Humano (HGMD), British Heart Foundation y bases de datos de Jojo Genetics.

Todas las muestras se analizaron mediante MLPA (Multiple Ligation-depend Probe Amplification) (MRC-Holland) para determinar la variación del número de copias ${ }^{15}$. La interpretación y clasificación de las variantes se estudiaron siguiendo las recomendaciones del consenso de la Asociación de Patología Molecular y del Colegio Médico Americano de Genética y Genoma (ACMG).

\section{Análisis estadístico}

Se utilizó el software SPSS versión 24. Las variables numéricas fueron representadas por la media, la desviación estándar y sus cuartiles. Las variables cualitativas fueron representadas por su frecuencia y porcentaje. Se utilizó la prueba t de
Student para la comparación de medias de grupos independientes y la prueba U de Mann-Whitney para la comparación de medianas. Se verificó la normalidad de las variables mediante la prueba de Shapiro-Wilk. En todos los casos, se utilizó un nivel de significancia de 0,05 .

\section{Resultados}

La población en estudio incluyó 55 casos índices de niños y adolescentes, 32 de sexo femenino y 23 de sexo masculino, de edad promedio de 9,5 $\pm 3,6$ años, de los cuales $77 \%$ consultó por dislipidemia y $23 \%$ fue reclutado por contar con el antecedente de ECV precoz demostrada.

Se identificaron 17 portadores de mutaciones asociadas a HF $(30,9 \%)$ y en 38 de ellos $(69,1 \%)$ no se identificó ninguna mutación. Los promedios de edad, IMC y concentraciones plasmáticas de lípidos se presentan en la Tabla 1. La media de CT y C-LDL fueron significativamente superiores en el grupo portador de mutaciones asociadas a $\mathrm{HF}$ comparado con el grupo sin mutación (CT, 308 $\pm 73 \mathrm{mg} / \mathrm{dL}$ vs. $213 \pm 34 \mathrm{mg} / \mathrm{dL}$ respectivamente; $\mathrm{p}=0,0001 ; \mathrm{y}$ C-LDL $238 \pm 75 \mathrm{mg} / \mathrm{dL}$ vs. $137 \pm$ $33 \mathrm{mg} / \mathrm{dL}$, respectivamente; $\mathrm{p}<0,0001)$. Ambos grupos no presentaron diferencias significativas en las medias de la edad ni en las medianas del IMC. Además, no se encontró diferencias estadísticamente significativas entre las medias de C-HDL $(\mathrm{p}=0,5271)$ y entre las medianas de TG $(\mathrm{p}=0,0718)$ de ambos grupos.

Al considerar los análisis genéticos de los

Tabla 1. Características clínicas y perfil lipídico de niños y adolescentes con y sin mutaciones asociadas a Hipercolesterolemia Familiar

\begin{tabular}{|c|c|c|c|c|c|c|c|c|c|c|c|}
\hline \multirow[b]{2}{*}{ Variable } & \multicolumn{5}{|c|}{ Con mutaciones $(n=17)$} & \multicolumn{5}{|c|}{ Sin mutaciones $(n=38)$} & \multirow[b]{2}{*}{ Valor $\mathbf{p}$} \\
\hline & Media & DE & Mediana & Q1 & Q3 & Media & DE & Mediana & Q1 & Q3 & \\
\hline 'Edad (años) & 8,9 & 4,2 & 9,0 & 6,0 & 10,0 & 9,7 & 3,3 & 10,0 & 8,0 & 11,0 & 0,4197 \\
\hline MWIMC (kg/m²) & 19,3 & 5,3 & 17,0 & 15,1 & 21,6 & 20,8 & 4,1 & 20,3 & 17,2 & 22,9 & 0,0778 \\
\hline${ }^{\mathrm{T}} \mathrm{CT}(\mathrm{mg} / \mathrm{dL})$ & 308 & 73 & 325 & 243 & 348 & 213 & 34 & 215 & 191 & 234 & 0,0001 \\
\hline${ }^{\top} \mathrm{LDL}-\mathrm{C}(\mathrm{mg} / \mathrm{dL})$ & 238 & 75 & 260 & 174 & 279 & 137 & 33 & 135 & 120 & 158 & $<0,0001$ \\
\hline${ }^{\mathrm{T}} \mathrm{HDL}-\mathrm{C}(\mathrm{mg} / \mathrm{dL})$ & 54 & 11 & 52 & 44 & 63 & 56 & 11 & 55 & 50 & 64 & 0,5271 \\
\hline MWTG (mg/dL) & 80 & 35 & 74 & 55 & 99 & 104 & 48 & 90 & 66 & 122 & 0,0718 \\
\hline
\end{tabular}

Tt-Student, MW Mann-Whitney, DE: desviación estándar, Q1 cuartil 1 y Q3 cuartil 3 
A

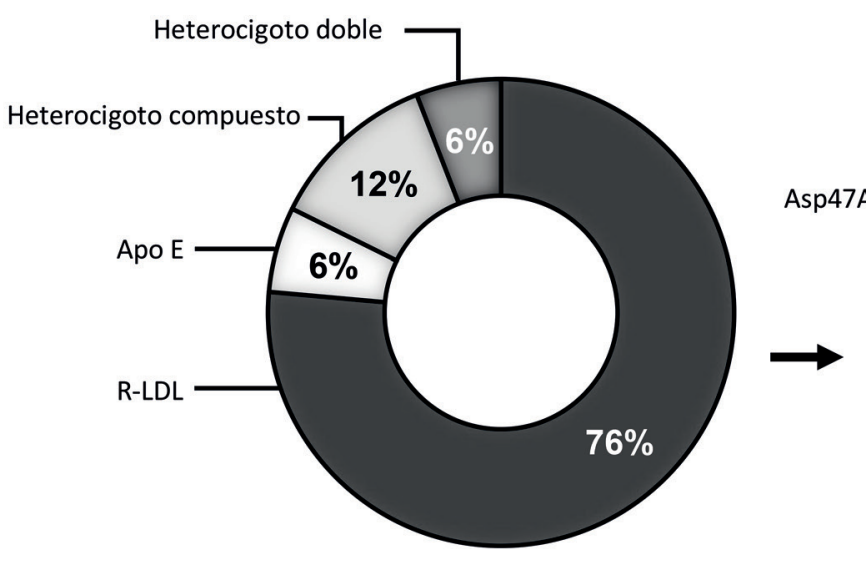

B

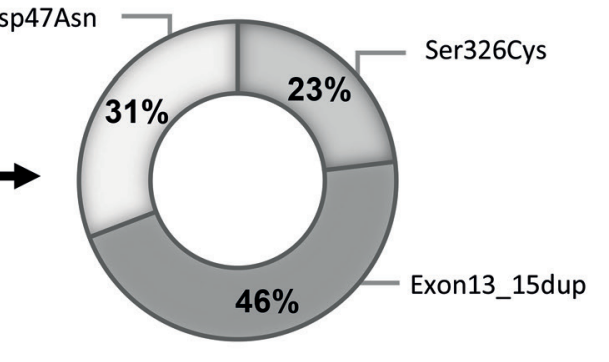

Figura 1. Distribución porcentual de las mutaciones asociadas a Hipercolesterolemia Familiar identificadas en la población de niños y adolescentes. (A) Porcentaje de los genes con variantes genéticas y de los genotipos compuestos y dobles. (B) Mutaciones en el R-LDL.

casos índices de niños y adolescentes con mutaciones asociadas a HF, se observó que 13 casos presentaron sólo una mutación en el gen del $R L D L$ y 1 caso tenía una mutación en el gen de la $A P O E$. Además, 2 casos son heterocigotos compuestos con las mutaciones p.Asp47Asn y duplicación de exones 13-15 del gen de $R L D L$ (Exon13_15dup), y uno es heterocigoto doble, portador de una duplicación de exones 13-15 en el gen $R L D L$ y la p.Ile268Met en el gen de la $A P O E$ (Figura 1A). Las variantes encontradas en el $R L D \mathrm{~L}$ correspondieron a: 3 heterocigotos para la variante p.Ser326Cys, 3 heterocigotos y un homocigoto para la mutación p.Asp47Asn y 6 heterocigotos con duplicación de exones 13-15 (Figura 1B). Además, se identificó sólo un niño homocigoto para la mutación p.Glu204* en el gen de la APOE (Tabla 2).

El análisis del perfil lipídico de los niños y adolescentes portadores de mutaciones asociadas a HF, mostró que los heterocigotos compuestos y los dobles presentaron niveles de CT y C-LDL mayores a $300 \mathrm{mg} / \mathrm{dL}$ y los portadores de la duplicación de exones 13-15 presentaron concentraciones promedio de CT y C-LDL de $304 \pm 70 \mathrm{mg} / \mathrm{dL}$ y $240 \pm 67 \mathrm{mg} / \mathrm{dL}$, respectivamente, valores que son superiores a los encontrados en las otras dos mutaciones del $R L D L$. Se observó la presencia de xantomas en un niño de genotipo heterocigoto para la duplicación de exones 13-15 con niveles de C-LDL de $240 \mathrm{mg} / \mathrm{dL}$ y en un heterocigoto compuesto que presentó concentración de C-LDL de $353 \mathrm{mg} / \mathrm{dL}$. Respecto a la mutación p.Glu204* del gen de la ApoE de genotipo homocigoto, se destaca las altas concentraciones de CT y C-LDL, niveles que son superiores a $250 \mathrm{mg} / \mathrm{dL}$ (Tabla 2). Las bases de datos consultadas para las variantes genéticas del R-LDL, establecen alelos patogénicos y de patogenicidad incierta para las distintas mutaciones identificadas en este estudio. Para las variantes derivadas de la ApoE, aunque no se encuentran definidas en las bases de datos, son consideradas como variantes de patogenicidad incierta (Tabla 2).

En la Figura 2, se presenta la localización de las mutaciones en el RLDL, observándose que la p.Asp47Asn se encuentra en el dominio de unión a ligando, la p.Ser326Cys en el dominio tipo EGF-1 (Epidermal Growth Factor-1) y la duplicación de exones 13-15 afecta tanto a la región de EGF-1 como al dominio de O-glicosilación de la proteína. Con respecto a la $A P O E$, la mutación p.Glu204* se encuentra en una región denominada intrínsecamente desordenada (IDR) del dominio bisagra y la variante p.Ile268Met en el extremo C-terminal de la proteína ${ }^{16}$. 
Hipercolesterolemia familiar: variantes genéticas en pediatría - A. Sánchez et al

Tabla 2. Identificación de mutaciones asociadas a Hipercolesterolemia Familiar, concentraciones plasmáticas de lípidos, presencia de xantomas y clasificación de su patogenicidad

\begin{tabular}{|c|c|c|c|c|c|c|c|c|}
\hline \multirow[t]{2}{*}{ Mutaciones } & \multirow[t]{2}{*}{$\mathbf{n}$} & \multicolumn{4}{|c|}{ Perfil lipídico (mg/dL) } & \multirow[t]{2}{*}{$\begin{array}{l}\text { Xan- } \\
\text { tomas }\end{array}$} & \multicolumn{2}{|c|}{$\begin{array}{c}\text { Clasificación según base } \\
\text { de datos }\end{array}$} \\
\hline & & CT & LDL-C & HDL-C & TG & & Clin Var & gnomAD \\
\hline \multicolumn{9}{|l|}{ R-LDL } \\
\hline p.Ser326Cys & 3 & $281 \pm 55$ & $216 \pm 44$ & $56 \pm 13$ & $46 \pm 8$ & & Patogénica & Patogénica \\
\hline p.Asp47Asn & 4 & $252 \pm 54$ & $174 \pm 70$ & $59 \pm 11$ & $95 \pm 32$ & & $\begin{array}{l}\text { Probablemente } \\
\text { patogénica; VUS }\end{array}$ & $\begin{array}{c}\text { Probablemente } \\
\text { deletérea }\end{array}$ \\
\hline Exon13_15dup & 6 & $304 \pm 70$ & $240 \pm 67$ & $49 \pm 8$ & $80 \pm 32$ & Sí & $\begin{array}{l}\text { Patogénica; } \\
\text { VUS }\end{array}$ & Ausente \\
\hline Exon13_15dup + p.Asp47Asn & 2 & $422 \pm 6$ & $353 \pm 17$ & $47 \pm 11$ & $109 \pm 64$ & Sí & & \\
\hline \multicolumn{9}{|l|}{ ApoE } \\
\hline p.Glu204* & 1 & 348 & 259 & 70 & 95 & & Ausente & Ausente \\
\hline $\begin{array}{l}\text { Exon13_15dup (R-LDL) + } \\
\text { p.lle268Met (apoE) }\end{array}$ & 1 & 370 & 307 & 52 & 55 & & $\begin{array}{l}\text { I268M: } \\
\text { ausente }\end{array}$ & $\begin{array}{l}\text { I268M: } \\
\text { ausente }\end{array}$ \\
\hline
\end{tabular}

VUS: variant uncertain significance.

A



R-LDL
Dominio de unión a ligando

Dominio de homología del precursor de EGF

Dominio de O-glicosilación

Dominio transmembrana

Dominio citoplasmático

B

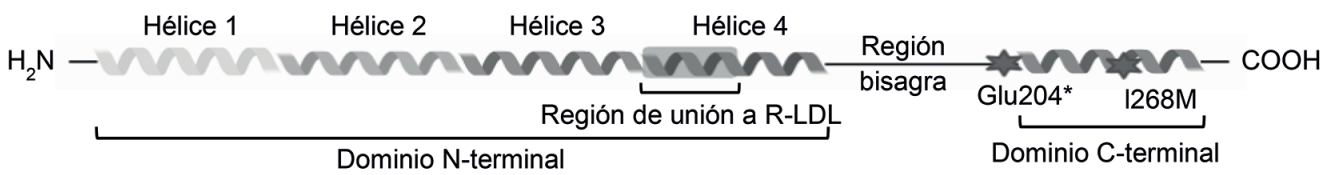

ApoE

Figura 2. Esquema con la localización de las mutaciones identificadas. (A) Receptor de LDL y (B) ApoE. Figuras realizadas en biorender.com. 


\section{Discusión}

La HF es una enfermedad autosómica dominante que se relaciona con el desarrollo de aterosclerosis temprana desde la infancia. En Chile, la información acerca de variantes genéticas asociadas a HF es escasa ${ }^{11}$ y no existe un programa sistemático de pesquisa de esta enfermedad. En este estudio se identificó las variantes genéticas: duplicación exones 13-15, p.Ser326Cys y p.Asp47Asn en el gen del $R L D L$; p.Glu204* y p.Ile268Met en el gen de $A P O E$, en población pediátrica con sospecha diagnóstica de HF, aportando a la caracterización genética de esta patología en nuestro país.

En la población estudiada de niños y adolescentes se identificó a 17 portadores de mutaciones asociadas a HF, los que mostraban niveles significativamente más altos de CT y C-LDL en comparación a aquellos que no presentaban mutación. La determinación de las concentraciones de CT y C-LDL están en corcordancia con estudios enfocados a detectar la HF en edad infanto-juvenil, los cuales señalan que estos parámetros lipídicos contribuyen a la detección de posibles portadores de $\mathrm{HF}^{17,18}$. Aunque el diagnóstico clínico actual se basa fundamentalmente en los niveles de C-LDL, el fenotipo de HF en población pediátrica diagnosticada genéticamente es diverso, con valores de C-LDL que fluctúan en un amplio rango ${ }^{17}$. Ante la sospecha de HF, se recomienda la pesquisa selectiva a partir de los 2 años de edad.

En relación a la frecuencia de las mutaciones descritas, se observó que el mayor porcentaje de ellas afectan al gen del $R L D L$, lo cual está en concordancia a lo publicado por diversos autores que señalan que más del 70\% de los casos de HF se asocian a mutaciones en este gen $^{4-6,19}$. En el presente estudio la duplicación de exones 13-15 resultó ser la alteración genética más frecuente identificada en la población. Si bien, esta mutación produce un reordenamiento génico mayor, existe poca información sobre el efecto de esta duplicación en la estructura y función del receptor. Sin embargo, se ha sugerido que esta variante genética produciría un codón de término anticipado de la transcripción, llevando a la expresión de un receptor carente del dominio de unión a membrana y citoplasmático $y$, por ende, no presente en la superficie celular ${ }^{20}$.

La segunda mutación más frecuente en este estudio fue la p.Asp47Asn (30,8\%), variante des- crita principalmente en población japonesa ${ }^{21} \mathrm{y}$ que no ha sido reportada previamente en Latinoamérica ${ }^{11}$. Esta mutación se localiza en la primera región abundante en cisteína (LA1) en el dominio $\mathrm{N}$-terminal involucrado en la unión al ligando del R-LDL (Figura 2a). Esta variante genética ha sido clasificada de patogenicidad incierta o probablemente patogénica por distintas bases de datos. En este contexto, nuestro grupo de investigación analizó 3 casos índices pediátricos portadores de esta mutación, los cuales presentaron valores CT y C-LDL significativamente más altos que los niños y adolescentes sin mutación, sugiriendo que la variante p.Asp47Asn podría tener un efecto deletéreo sobre el metabolismo del colesterol (datos no publicados).

Respecto a la mutación patogénica p.Ser326 Cys, se encontró en un 23,1\% de los casos estudiados, lo cual coincide con lo señalado en la literatura que posiciona a esta variante como una de las más prevalentes en Chile y en otros países latinoamericanos como Brasil ${ }^{11}$. Esta mutación se encuentra en el dominio altamente conservado EGF-1, el cual está implicado en el reciclaje del receptor hacia la membrana y en la liberación de receptor dentro del endosoma, por lo que defectos en este dominio tienen como consecuencia una mayor degradación del receptor y menor expresión del receptor en la superficie celular ${ }^{22,23}$.

Existe poca evidencia en la literatura que relacione mutaciones en el gen de APOE con la HF. En este estudio se identificaron 2 mutaciones en este gen: p.Glu204* y p.Ile268Met. En el homocigoto para p.Glu $204^{\star}$ se encontró que los niveles de CT y C-LDL fueron considerablemente superiores a los valores de referencia. Si bien la ApoE es estructuralmente compleja, se ha demostrado que cambios en un solo aminoácido producen un efecto global que compromete toda la estructura de la proteína ${ }^{24}$. Para llevar a cabo su función, la ApoE debe ser estructuralmente flexible para que ocurran los cambios conformacionales requeridos para la unión de lípidos a sus dominios $\mathrm{N}$ y C-terminal ${ }^{16,24}$; por lo tanto, la mutación p.Glu204* y la variante p.Ile268Met podrían alterar la interacción de ApoE con lípidos.

La información de los fenotipos de los portadores heterocigotos compuestos (dos mutaciones asociadas a HF en el mismo gen) y heterocigotos dobles (dos variantes asociadas a HF en genes distintos) es escasa ${ }^{25}$. En este contexto, el Panel de 
Consenso de la Sociedad Europea de Aterosclerosis declaró que se encuentran menores niveles de C-LDL en los heterocigotos dobles en comparación con los portadores homocigotos de mutaciones en $A P O B, P C S K 9$ o $R L D L^{26}$. Más aún, se ha reportado que los valores promedio de C-LDL en los heterocigotos dobles se encuentran entre los niveles observados en los portadores heterocigotos y heterocigotos compuestos, pudiéndose asociar con una forma grave de HF heterocigota ${ }^{25-27}$. Así, los heterocigotos dobles y compuestos descritos en este estudio tuvieron niveles de C-LDL muy superiores a los presentados por los portadores heterocigotos para la duplicación de exones 13$15^{25-27}$.

Este estudio constituye un aporte a la caracterización de los genes asociados a HF en una población de niños y adolescentes chilenos. Sin embargo, contempló un número acotado de participantes de la comuna de Concepción y representa la etapa inicial de la pesquisa de esta patología, cuya continuidad permitirá avanzar en el conocimiento de la realidad de la HF en nuestra población.

En conclusión, se logró identificar portadores de mutaciones asociadas a $\mathrm{HF}$, describir las variantes genéticas y sus fenotipos a través de la pesquisa selectiva de niños y adolescentes con hipercolesterolemia y/o antecedentes familiares de ECV precoz demostrada en una población chilena.

\section{Referencias}

1. Defesche JC, et al. Familial hypercholesterolaemia. Nat Rev Dis Primers 2017; 3: 17093.

2. Mata P, et al. [Diagnosis and treatment of familial hypercholesterolemia in Spain: Consensus document]. Semergen 2015; 41 (1): 24-33.

3. Rubio-Marin $\mathrm{P}$, et al. Cascade screening program for familial hypercholesterolemia. Endocrinol Diabetes Nutr. 2018; 65 (5): 280-6.

4. Kastelein JJP, Reeskamp LF, Hovingh GK. Familial Hypercholesterolemia: The Most Common Monogenic Disorder in Humans. J Am Coll Cardiol. 2020; 75 (20): 2567-9.

5. Banares VG, et al. Preliminary spectrum of genetic variants in familial hypercholesterolemia in Argentina. J Clin Lipidol. 2017; 11 (2): 524-31.

6. Di Taranto MD, Giacobbe C, Fortunato G. Familial hypercholesterolemia: A complex genetic disease with variable phenotypes. Eur J Med Genet. 2020; 63 (4): 103831.

7. Wiegman A, et al. Familial hypercholesterolaemia in children and adolescents: gaining decades of life by optimizing detection and treatment. Eur Heart J. 2015; 36 (36): 2425-37.

8. McCrindle BW, Gidding SS. What Should Be the Screening Strategy for Familial Hypercholesterolemia? N Engl J Med. 2016; 375 (17): 1685-6.

9. $\mathrm{Ll}$ a, et al. Hipercolesterolemia familiar heterocigota: diagnóstico molecular y terapia combinada. Caso clínico. Revista Médica de Chile - Rev Med Chile 2007; 135.

10. Akioyamen LE, et al. Estimating the prevalence of heterozygous familial hypercholesterolaemia: a systematic review and meta-analysis. BMJ Open. 2017; 7 (9): e016461.

11. Santos RD, et al. Clinical and molecular aspects of familial hypercholesterolemia in Ibero-American countries. J Clin Lipidol. 2017; 11 (1): 160-6.

12. Alves AC, et al. Phenotypical, Clinical, and Molecular Aspects of Adults and Children With Homozygous Familial Hypercholesterolemia in Iberoamerica. Arterioscler Thromb Vasc Biol. 2020; 40 (10): 2508-15.

13. Centers for Disease Control and Prevention: CDC Growth Charts: U.S. Available from: http://www.cdc. gov/growthcharts/data_tables.

14. Friedewald WT, Levy RI, Fredrickson DS. Estimation of the concentration of low-density lipoprotein cholesterol in plasma, without use of the preparative ultracentrifuge. Clin Chem. 1972; 18 (6): 499-502.

15. Silva PRS, et al. Predictors of Family Enrollment in a Genetic Cascade Screening Program for Familial Hypercholesterolemia. Arq Bras Cardiol. 2018; 111 (4): 578-84.

16. Frieden C, Wang H, Ho CMW. A mechanism for lipid binding to apoE and the role of intrinsically disordered regions coupled to domain-domain interactions. Proc Natl Acad Sci USA 2017; 114 (24): 6292-7.

17. Plana N, et al. Lipid and lipoprotein parameters for detection of familial hypercholesterolemia in childhood. The DECOPIN Project. Clin Investig Arterioscler. 2018; 30 (4): p. 170-8.

18. Wald DS, Bestwick JP, Wald NJ. Child-parent screening for familial hypercholesterolaemia: screening strategy based on a meta-analysis. BMJ. 2007; 335 (7620): 599.

19. Nordestgaard BG, et al. Familial hypercholesterolaemia is underdiagnosed and undertreated in the general population: guidance for clinicians to prevent coronary heart disease: consensus statement of the European Atherosclerosis Society. Eur Heart J. 2013; 34 (45): 3478-90a. 
20. Lelli N, et al. Duplication of exons 13, 14 and 15 of the LDL-receptor gene in a patient with heterozygous familial hypercholesterolemia. Hum Genet. 1991; 86 (4): 359-62.

21. Varret M, et al. LDLR Database (second edition): new additions to the database and the software, and results of the first molecular analysis. Nucleic Acids Res. 1998; 26 (1): 248-52.

22. Arias-Moreno X, et al. Mechanism of low density lipoprotein (LDL) release in the endosome: implications of the stability and Ca2+ affinity of the fifth binding module of the LDL receptor. J Biol Chem. 2008; 283 (33): 22670-9.

23. Lu R, et al. Spontaneous severe hypercholesterolemia and atherosclerosis lesions in rabbits with deficiency of low-density lipoprotein receptor (LDLR) on exon 7.
EBioMedicine 2018; 36: 29-38.

24. Frieden C, Garai K. Concerning the structure of apoE. Protein Sci. 2013; 22 (12): 1820-5.

25. Sjouke B, et al. Double-heterozygous autosomal dominant hypercholesterolemia: Clinical characterization of an underreported disease. J Clin Lipidol. 2016: 10 (6): 1462-9.

26. Cuchel M, et al. Homozygous familial hypercholesterolaemia: new insights and guidance for clinicians to improve detection and clinical management. A position paper from the Consensus Panel on Familial Hypercholesterolaemia of the European Atherosclerosis Society. Eur Heart J. 2014; 35 (32): 2146-57.

27. Pamplona-Cunha H, et al. Compound Heterozygous Familial Hypercholesterolemia Caused by LDLR Variants. Arq Bras Cardiol 2020; 115 (3): 587-9. 